\title{
Attention deficits in Japanese multiple sclerosis patients with minor brain lesion loads
}

\author{
This article was published in the following Dove Press journal: \\ Neuropsychiatric Disease and Treatment \\ 15 December 201। \\ Number of times this article has been viewed
}

\author{
Akitoshi Takeda ${ }^{1,3}$ \\ Masashi Nakajima' \\ Mutsutaka Kobayakawa' \\ Natsuko Tsuruya' \\ Shin-ichi Koyama ${ }^{1,2}$ \\ Takami Miki ${ }^{3}$ \\ Mitsuru Kawamura ${ }^{1,4}$ \\ 'Department of Neurology, Showa \\ University School of Medicine, Tokyo, \\ Japan; ${ }^{2}$ Faculty of Engineering, Chiba \\ University, Chiba, Japan; ${ }^{3}$ Department \\ of Geriatric Medicine and Neurology, \\ Osaka City University Graduate \\ School of Medicine, Osaka, Japan; \\ ${ }^{4}$ Core Research for Evolutional \\ Science and Technology (CREST), \\ Japan Science and Technology Agency \\ (JST), Saitama, Japan
}

Background: To investigate whether Japanese multiple sclerosis (MS) patients with minor brain lesion loads have attention deficits and brain atrophy, and to correlate their circumstance.

Method: Twenty-one Japanese patients with relapsing-remitting MS were included in this study. Attention deficits were evaluated using Clinical Assessment for Attention (CAT) standardized according to age groups. Lesion load in the brain was assessed by tallying the total volume of plaques visible on brain magnetic resonance imaging (MRI). The width of the third ventricle and the bicaudate ratio were measured.

Results: The completion time for the visual cancellation tasks and/or the reaction times for the continuous performance test were prolonged in 14 patients $(66.7 \%)$. The accuracy of responses was preserved throughout the CAT. Deviation from the normal value was not exaggerated based on the increasing difficulty of the task. The total volume of plaques on brain MRI was small. The width of the third ventricle was significantly increased in patients with MS when compared to controls, but was not correlated with the low performance on the CAT.

Conclusions: Japanese MS patients with minor brain lesion loads frequently had attention deficits characterized by slowness of automatic information processing, but controlled processing that requires working memory demands was spared.

Keywords: attention, cognitive function, magnetic resonance imaging, multiple sclerosis

\section{Introduction}

In North American and European studies, cognitive impairment occurs in $40 \%-65 \%$ of patients with multiple sclerosis (MS). ${ }^{1}$ Cognitive decline appears in MS patients in the early stage of the disease and impacts social functioning and employment, ${ }^{2,3}$ but rarely progresses to dementia. ${ }^{4}$ Attention deficit may be an important indicator of cognitive decline ${ }^{3-5}$ and may occur in the early stages of the disease. ${ }^{3}$ Recent magnetic resonance imaging (MRI) studies revealed that cognitive impairment in MS is dependent on brain atrophy rather than on the total lesion load. ${ }^{6-8} \mathrm{MS}$ in Asians is characterized by a low prevalence rate, a preferential involvement of the optic nerve and the spinal cord, and few plaques in the brain. ${ }^{9}$ Whether Asian MS patients with less brain lesion burdens have cognitive impairment and brain atrophy, as is the case with MS patients in Western populations, has never been evaluated. We investigated cognitive impairment in Japanese relapsing-remitting MS patients with a focus on attention deficits and correlated them to MRI measures of brain atrophy.
Department Taked ment of Geriatrics and Neurology Osaka City University Graduate Schoo of Medicine, I-4-3 Asahimachi, Abeno-ku, Osaka 545-8585, Japan

Tel +8I 666453889

Fax +8I 666465599

Email a-taked@med.osaka-cu.ac.jp 


\section{Methods}

\section{Patients and control subjects}

Of 26 consecutive patients with clinically definite MS $^{10}$ who attended Showa University Hospital, 22 patients were classified as having relapsing-remitting MS. Excluding one patient who had a psychiatric illness, we included 21 patients (six men and 15 women) in this study. The study protocol was approved by the institutional ethics committee, and all patients gave written informed consent prior to participation. Demographic characteristics are summarized in Table 1. The level of physical disability was measured with the Expanded Disability Status Scale (EDSS). ${ }^{11}$ EDSS scores ranged from 1.0 to 5.0. Significant disabilities were limited to lower limb functions, and all the patients had well-preserved upper limb and visual functions (EDSS Functional System grade $\leq 2$ ). Physical disabilities in most patients were attributed to spinal cord or brain stem lesions. No patient fulfilled the diagnostic criteria of neuromyelitis optica. ${ }^{12}$ One patient (patient 3) tested positive for the serum anti-aquaporin 4 channel antibody.

\section{Assessment of attention deficits}

Attention deficits were assessed systematically using the Clinical Assessment for Attention (CAT) developed and standardized by the Japan Society for Higher Brain Dysfunction. ${ }^{13}$
CAT assesses attention with respect to reaction time, vigilance, short-term storage capacity, mental tracking (working memory), and complex attention. ${ }^{14}$ Individual tasks include digit and visual tapping spans, a visual cancellation task, an auditory detection task, ${ }^{15}$ a symbol digit modalities test (SDMT) ${ }^{16}$ a memory-updating test, ${ }^{17}$ a paced auditory serial addition test (PASAT), ${ }^{18}$ a position Stroop test, ${ }^{19}$ and a continuous performance test (CPT) ${ }^{20}$

The digit span test consists of digits forward and digits backward and was given to the subjects in the manner of the Wechsler batteries. The visual tapping span test uses the Corsi block-tapping task, and subjects were asked to copy the tapping pattern forward and backward following the examiner's taps in the same prearranged sequence as the digit span test. The visual cancellation task is a simple test measuring processing speed and accuracy. Using a pencil, subjects were asked to cross out a target stimulus dispersed within rows of random, interfering stimuli displayed on a sheet. Two sets of the stimulus sequence were used: digits (part I) and kana-letters (part II). The time required to complete the task (completion time) and the ratio of correct answers to the total number of stimuli were assessed. The auditory detection task is a continuous performance test that presents a series of kana-letters read at the rate of one per second on a compact disc. Subjects were asked to tap their

Table I Demographics of patients and results of CAT and MRI measurements

\begin{tabular}{|c|c|c|c|c|c|c|c|c|c|}
\hline \multirow{2}{*}{$\begin{array}{l}\text { Patient number, } \\
\text { age/sex }\end{array}$} & \multirow{2}{*}{$\begin{array}{l}\text { Duration } \\
\text { (months) }\end{array}$} & \multirow{2}{*}{$\begin{array}{l}\text { Relapse } \\
\text { (number) }\end{array}$} & \multirow[t]{2}{*}{ EDSS } & \multirow[t]{2}{*}{ MMSE } & \multirow[t]{2}{*}{ CAT* } & \multicolumn{2}{|c|}{ MRI lesions } & \multicolumn{2}{|c|}{ MRI measures } \\
\hline & & & & & & Brain** & Others & WTV & BCR \\
\hline $\mathrm{I}, 45 / \mathrm{F}$ & 96.5 & 4 & 4.0 & 30 & 3 & 0.09 & BS, SC & - & - \\
\hline $2,4 \mathrm{I} / \mathrm{F}$ & 18.8 & I & 2.5 & 30 & 2 & 0.31 & BS & 3.73 & 0.12 \\
\hline $3,27 / M$ & 96.9 & 2 & 1.0 & 30 & 8 & 0.00 & BS & 6.59 & 0.17 \\
\hline $4,39 / F$ & 144.4 & 7 & 2.0 & 30 & 5 & 0.04 & ON & 4.59 & 0.12 \\
\hline $5,56 / F$ & 4.3 & 2 & 2.5 & 29 & 12 & 3.42 & None & 4.59 & 0.15 \\
\hline $6,30 / F$ & 48.6 & 4 & 1.5 & 30 & 4 & 0.00 & BS & 4.87 & 0.15 \\
\hline $7,25 / M$ & 6.8 & I & 2.0 & 30 & 6 & 0.88 & $\mathrm{SC}, \mathrm{ON}$ & - & - \\
\hline $8,34 / F$ & 72.7 & 8 & 3.5 & 26 & 4 & 0.21 & SC & 3.73 & 0.14 \\
\hline $9,44 / F$ & 300.4 & 8 & 2.0 & 30 & 2 & 0.04 & ON & 6.59 & 0.14 \\
\hline $10,55 / F$ & 360.7 & 6 & 5.0 & 30 & I & 1.98 & SC & 6.59 & 0.15 \\
\hline $\mathrm{II}, 50 / \mathrm{F}$ & 192.8 & 4 & 1.5 & 30 & 0 & 0.14 & BS, SC & 6.31 & 0.16 \\
\hline $12,47 / M$ & 300.3 & 5 & 1.5 & 30 & I & 0.57 & SC & 7.74 & 0.17 \\
\hline $13,50 / F$ & 156.2 & 4 & 1.0 & 30 & 5 & 0.07 & SC & - & - \\
\hline $14,35 / M$ & 1.5 & I & 1.0 & 29 & 5 & 0.54 & SC & 2.29 & 0.12 \\
\hline $15,33 / F$ & 8.6 & I & 2.5 & 30 & 1 & 0.06 & BS & 6.59 & 0.13 \\
\hline $16,42 / M$ & 1.3 & I & 1.5 & 27 & 6 & 0.17 & BS, SC & 4.3 & 0.14 \\
\hline I7, 28/F & 1.5 & I & 2.5 & 30 & 8 & 1.94 & None & 4.87 & 0.15 \\
\hline $18,36 / F$ & 4.9 & I & 2.0 & 30 & 2 & 0.00 & SC & - & - \\
\hline $19,20 / \mathrm{M}$ & 1.9 & I & 2.5 & 30 & 3 & 1.69 & SC & 3.73 & 0.11 \\
\hline $20,4 \mathrm{I} / \mathrm{F}$ & 5.0 & I & 2.0 & 27 & I & 0.06 & SC & 7.74 & 0.14 \\
\hline $21,42 / F$ & 1.6 & I & 2.0 & 28 & 7 & 0.18 & SC & - & - \\
\hline
\end{tabular}

Notes: *Number of CAT assessments with a $Z$ score $<-1.5 \mathrm{SD}$; **Total lesion load $(\mathrm{mL})$; -, not examined.

Abbreviations: M, male; F, female; EDSS, Expanded Disability Status Scale; MMSE, Mini-mental status examination; CAT, Clinical Assessment for Attention; MRI, magnetic resonance imaging; WTV, width of third ventricle $(\mathrm{mm})$; BCR, bicaudate ratio; BS, brain stem; SC, spinal cord; ON, optic nerve. 
finger each time they heard the target letter. Increasing the phonetic similarity between the target and background stimuli heightens the task difficulty. Both the ratio of correct answers and the accuracy (the number of accurate answers compared to the number of total responses) were assessed. The SDMT is a visuographic task that preserves the substitution format of Wechsler's Digit Symbol test but reverses the presentation of the material so that symbols are printed and numbers are written out. Ninety seconds were allowed for each trial, which contained 110 items. The ratio of completion (the number of correct answers compared to the number of items) was assessed. The memory-updating test requires subjects to listen to strings of digits of unknown length from the subject's perspective and then to recall the three or four most recent digits. This means that subjects were required to remember the first three or four items presented and then, if there were more than three or four items in a list, to update the contents of their memory by dropping the oldest item and adding the most recent to the string. The PASAT requires the subjects to add 60 pairs of randomized digits so that each is added to the digit immediately preceding it. The digits were presented either every 1 or 2 seconds. The ratio of correct answers was assessed in each test. The position Stroop test uses a Japanese-kanji version of the High-Mid-Low format developed by Sohlberg. ${ }^{19}$ Subjects were asked to call out the position of kanji that mean high, mid, or low, instead of their meaning. One hundred and fourteen items were used in the trials, and were presented in six rows. The completion time and the ratio of correct answers were assessed. The CPT is a computerized vigilance test that presents stimuli briefly and provides reaction times as well as accuracy data. In the simple version, subjects responded to the digit "7" that appeared briefly in the center of the screen at random intervals. In the $\mathrm{X}$ version, digits appeared in random order, and subjects were asked to respond to every "7". In the more difficult (AX) version, subjects were asked to respond to "7" only if it followed "3". Each subject's reaction time (RT), ratio of correct answers, and accuracy were assessed for each test.

Several institutes associated with the Japan Society for Higher Brain Dysfunction studied 297 volunteers who had neither a history of brain damage nor significant medical conditions and who ranged in age from 20 to 79 years..$^{13}$ The mean and standard deviation (SD) for each assessment item, except for the CPT, were calculated for patients in each decade of life from their twenties to their seventies, with more than 40 subject numbers for each decade. The CPT was studied in 109 volunteers ranging in age from 23 to 79 years, and the normal value was calculated for patients in each decade of life from their twenties through their fifties $(n=52)$. A $Z$ score was calculated for each assessment item for individual patients. It was derived from the normal value of the corresponding age group and was defined as abnormal if it was $<-1.5 \mathrm{SD}$.

\section{MRI and measurements}

All patients underwent MRI of the brain and the spinal cord using a Siemens Magnetom Vision operating at 1.5 tesla (Siemens, Erlangen, Germany). The brain MR protocol included transverse T2-weighted images [repetition time/ echo time: 3800/96 milliseconds (ms)] with $5 \mathrm{~mm}$ thick contiguous slices and coronal fluid-attenuated inversion recovery (FLAIR) images (repetition time/echo time/ inversion time: $7000 / 110 / 2000 \mathrm{~ms}$ ) with $6 \mathrm{~mm}$ thick contiguous slices. For the spinal cord MRI, T2-weighted, and T1-weighted images were obtained in the sagittal and axial planes. MRI measurements were analyzed by a neurologist blinded to the patients' identity using Java Image software (Sun Microsystems, available at: http://rsb.info.nih.gov/ij/). White matter lesion load was calculated from T2-weighted images using a semiautomated segmentation technique. ${ }^{21,22}$ The width of the third ventricle (WTV) and the bicaudate ratio $(\mathrm{BCR})^{7,8,23}$ were measured from coronal FLAIR images in 16 patients and 13 gender- and age-matched controls. To obtain WTV, a line was drawn through the long axis of the ventricle parallel to the interhemispheric fissure in the section where the third ventricle was most visible. The width was measured by drawing a second line perpendicular to the first at its midpoint. The BCR was the minimum intercaudate distance divided by brain width along the same line and was measured in the slice where the heads of the caudate nuclei were most visible and closest to one another.

\section{Statistical analysis}

Nonparametric statistics were used. Differences between groups were tested using the Mann-Whitney $U$ test. Spearman's rank correlation coefficient was used to explore correlations between variables. Two-tailed tests to measure significance were used throughout. SPSS software (v 10.0; SPSS Inc, Chicago, IL) was used for the analysis. To rank the degree of attention deficits, we did not employ crude data of each assessment in the CAT but instead used the number of abnormal assessment items to exclude the effects of age.

\section{Results \\ Performance on CAT}

The number of low performance items with a $Z$ score $<-1.5 \mathrm{SD}$ in patients ranged from zero to twelve (mean, 4.1; SD, 3.0). The 
number of abnormal items did not correlate with any parameters, including age, duration of illness, number of relapses, EDSS, or mini-mental status examination (MMSE). With respect to individual assessment items on CAT (Table 2), completion time of the visual cancellation task and RTs for CPT, PASAT, and SDMT were frequently abnormal. In contrast, digit and tapping spans either forward or backward, the memory updating test, the percentage of correct answers on the visual cancellation, the auditory detection tasks, and CPT, and both the ratio of correct answer and the completion time of position Stroop test were relatively spared or impaired in a small number of patients. Because a completion time is equivalent to a sum of RTs, lengthening of RT was the most prominent abnormality found by CAT. A prolonged completion time for the visual cancellation task was the prevailing abnormality in our patients. However, most patients maintained the normal level of accuracy on this task. The completion time for the visual cancellation tasks and/or the RT for CPT was prolonged in 14 patients (66.7\%).

Table 2 Results of clinical assessment for attention

\begin{tabular}{|c|c|c|}
\hline Task & Assessment & $\begin{array}{l}\text { Patients }(\mathrm{n}) \text { with } \\
Z \text { score }<-I .5 \mathrm{SD}\end{array}$ \\
\hline Digit, forward & Span & I \\
\hline Digit, backward & Span & 2 \\
\hline Tapping, forward & Span & 3 \\
\hline Tapping, backward & Span & 4 \\
\hline \multicolumn{3}{|l|}{ Visual cancellation } \\
\hline \multirow[t]{2}{*}{ Part I, “3” } & $\%$ correct answer & 1 \\
\hline & Completion time & II \\
\hline \multirow[t]{2}{*}{ Part 2, “か”* } & $\%$ correct answer & $\mathrm{I}$ \\
\hline & Completion time & 6 \\
\hline \multirow[t]{2}{*}{ Auditory detection } & $\%$ correct answer & 4 \\
\hline & Accuracy & 3 \\
\hline $\begin{array}{l}\text { Symbol digit } \\
\text { modalities test }\end{array}$ & Achievement rate & 8 \\
\hline \multicolumn{3}{|l|}{ Memory updating test } \\
\hline 3 digits & $\%$ correct answer & 4 \\
\hline 4 digits & $\%$ correct answer & 3 \\
\hline \multicolumn{3}{|c|}{ Paced auditory serial addition test } \\
\hline $2 \mathrm{sec}$ & $\%$ correct answer & 6 \\
\hline I sec & $\%$ correct answer & 5 \\
\hline \multirow[t]{2}{*}{ Position stroop test } & $\%$ correct answer & 3 \\
\hline & Completion time & 2 \\
\hline \multicolumn{3}{|c|}{ Continuous performance test } \\
\hline \multirow[t]{3}{*}{ Simple version } & $\%$ correct answer & 0 \\
\hline & Accuracy & 0 \\
\hline & Reaction time & 6 \\
\hline \multirow[t]{3}{*}{$X$ version } & $\%$ correct answer & 2 \\
\hline & Accuracy & 2 \\
\hline & Reaction time & 4 \\
\hline \multirow[t]{3}{*}{$A X$ version } & $\%$ correct answer & 0 \\
\hline & Accuracy & 2 \\
\hline & Reaction time & 3 \\
\hline
\end{tabular}

Note: *One of Japanese kana-letters used as a target stimulus. Abbreviation: SD, standard deviation.
We correlated the deviation between tasks or items. CAT has an advantage in examining individuals' deviations from normal values in each assessment, excluding the effects of age. In CPT, the RT was not prolonged disproportionately with increasing task complexity. The number of patients with a $Z$ score $<-1.5 \mathrm{SD}$ was six in the simple version, four in the $\mathrm{X}$ version, and three in the AX version (Table 2). RTs for the simple and the $X$ or AX versions correlated positively, but the $Z$ score was smaller for the simple version RT compared to the $\mathrm{X}$ or AX versions (Figure 1). The ratio of correct answers and the accuracy of responses were well preserved throughout the CPT. The number of patients with a $Z$ score $<-1.5 \mathrm{SD}$ was nine $(42.9 \%)$ in either version of the PASAT, and five $(23.8 \%)$ in either version of the memory updating task. In each test, the $Z$ scores for the two versions correlated with increasing difficulty, and there was no further decline associated with increasing difficulty. The $Z$ score for individual patients was lower for the 2-second interval version than the 1-second interval version for PASAT and was lower in the three digits version compared to the four digits version for the memory-updating test (Figure 2). The achievement rates for SDMT and the completion times for the position Stroop test correlated positively to the completion times of the visual cancellation test (Figure 3 ). The $Z$ scores were lower in the visual cancellation task than in SDMT or the position Stroop test. The number of patients with a $Z$ score $<-1.5 \mathrm{SD}$ was eight (38.1\%) for SDMT, and four $(19.0 \%)$ for the position Stroop test.

\section{MRI and measurements}

White matter lesion load ranged from 0.0 to $3.42 \mathrm{~mL}$ (mean, 0.57; SD, 0.92; Table 1). The mean WTV was greater in patients $(5.3 \mathrm{~mm}$; SD, 1.6) than in controls ( $4.0 \mathrm{~mm}$; SD, 1.3; $P<0.05)$. There was no BCR difference between patients (mean, 0.14; SD, 0.02) and controls (mean, 0.12; SD, 0.02). In the patient group, there was no correlation between the number of low performance items in CAT or the $Z$ score of individual items and white matter lesion load, WTV, or BCR. The WTV correlated positively with duration of illness and BCR $(P<0.05)$, but not with the number of relapses or white matter lesion load.

\section{Discussion}

\section{Attention deficits in Japanese MS patients}

Using a standardized test that probes various domains of attention, we found that in Japanese relapsing-remitting MS patients slowed information processing was prevalent, 
CPT (SRT and XRT)

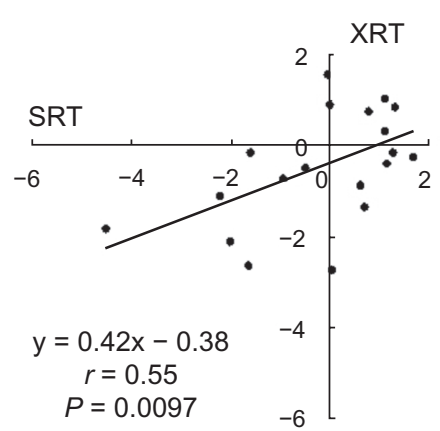

CPT (SRT and AXRT)

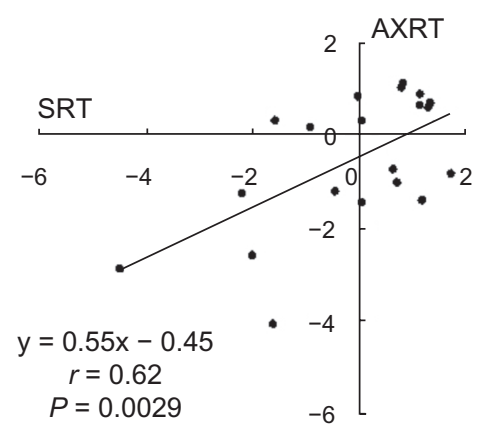

Figure I The correlations of individuals' $Z$ scores of the CPT between reaction times for simple (SRT) and X (XRT) (left), or AX versions (AXRT) (right) are represented. The individuals' $Z$ scores are lower for the simple version ( $x$-axis) than for the $X$ or AX versions ( $y$-axis).

whereas coherence to the task and accuracy of responses were preserved. Several factors including motor slowing, perceptual deficits, attention deficits, minor cognitive deficits, and overall mental slowing may account for this slowed information processing. ${ }^{24}$ In our patients, lengthening of the $\mathrm{RT}$ in CPT was larger for the simple version than for the tasks that required selective attention ( $\mathrm{X}$ and $\mathrm{AX}$ versions). This finding suggests that overall mental slowing or minor cognitive deficits are unlikely to be causing the slowed information processing in our patients because disproportionate lengthening of the RT with increasing difficulty has been reported in general slowness or cognitive impairment due to diffuse brain damage.$^{25}$ We were unable to exclude a slowing of motor acts and visual perceptions in this study, although our patients did not have significant deficits in motor function of the upper limbs or in visual acuity. However, we do not think that only motor or visual perceptual impairment contributes to the slowing of RT. Tasks that required information processing speed and were examined with auditory perception of stimuli, and verbal output, such as PASAT and the memory-updating test, were impaired as well (Figure 2).

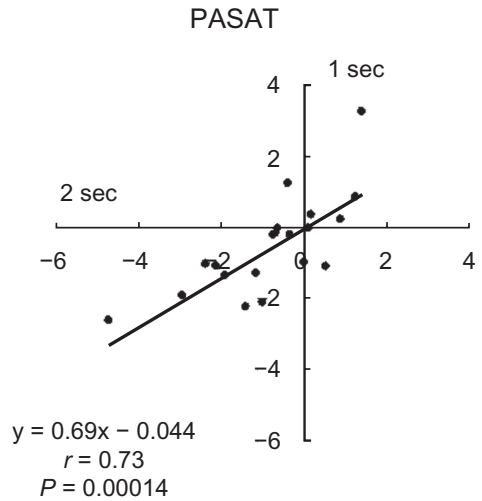

Kujala et al divided information processing into automatic and controlled processing in MS patients with or without cognitive deficits. ${ }^{5}$ They found that in cognitively preserved patients, automatic processing that requires relatively effortless recognition of simple visual stimuli was impaired, whereas controlled processing that demands working memory was preserved. In patients with mild cognitive deterioration, both automatic and controlled processing were impaired. The results of multiple tasks performed by our patients were comparable to the characteristics of information processing in the cognitively preserved patients. In PASAT and the memory-updating task, the increasing difficulty of the tasks was not accompanied by a decrease in performance (Figure 2). The deviations from normal values in SDMT and the position Stroop test were smaller than in the visual cancellation task (Figure 3). The position Stroop test requires not only selective attention, but also inhibition/suppression of distracting presentations. ${ }^{26}$ These results suggest that controlled information processing is preserved in spite of impaired automatic information processing. Although it is difficult to explain the even smaller deviation from normal

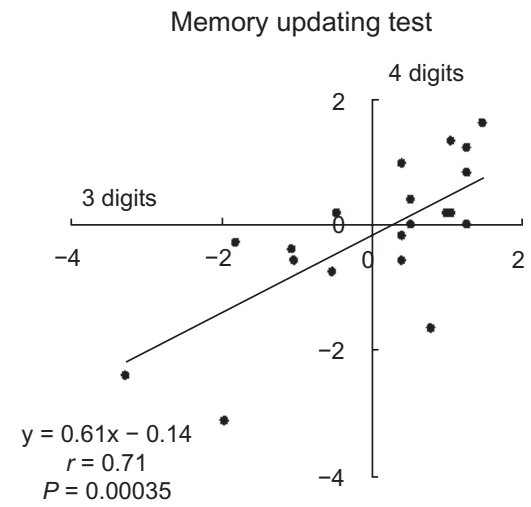

Figure 2 The correlation of individuals' $Z$ scores for two tests with increasing difficulty are represented: PASAT (left) and the memory-updating test (right). For PASAT, the individuals' $Z$ scores are lower for the 2-second version ( $x$-axis) than for the more difficult I-second version ( $y$-axis). For the memory-updating test, the individuals' $Z$ scores are lower for the three digits version (x-axis) than for the more difficult four digits version ( $y$-axis). 

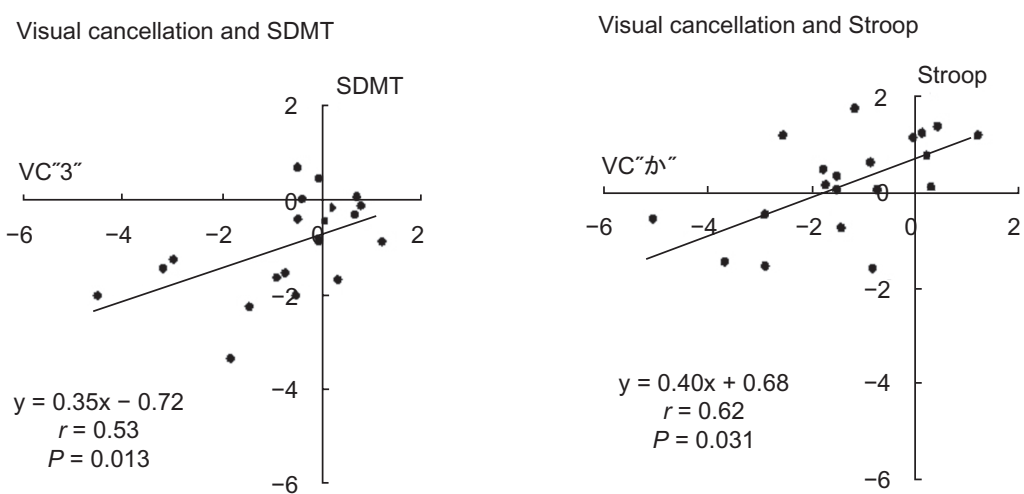

Figure 3 The correlation of individuals' Z scores between the visual cancellation task and SDMT (left), or position Stroop test (right) are represented. The individuals' $Z$ score is lower for the visual cancellation task (x-axis) than for the SDMT and the position Stroop test, which requires more elaboration of working memory ( $y$-axis).

with increasing difficulty of the task, we hypothesize that the further burden of working memory demands could have evoked the attentional system in our patients.

\section{Attention deficits and brain lesion load or atrophy}

In comparison with Western MS patients, our patients were clinically characterized by predominantly spinal cord or brain stem involvement. Lesion load in the brain, estimated from the total volume of plaques on MRI, was much less than that reported for European patients. ${ }^{21,22}$ Attention deficits were present in patients within months after onset of the clinical disease and were also present in patients who had no visible plaques in their brain. Furthermore, the degree of attention deficits measured by number of abnormal items from CAT did not correlate with the duration of illness, physical disabilities, or brain lesion score. These findings in Japanese MS patients with minor brain lesion load support the idea that a neurodegenerative process may be a pathological substrate of the attention deficits in MS. 6,27,28

In this study, we did not find any correlation between attention deficits and duration of illness, number of plaques, or MRI measures of brain atrophy. The WTV, one of the indices of brain atrophy, ${ }^{7,8}$ was increased in patients and correlated positively to illness duration but not to the number of low performance items from CAT. This finding is contrary to previous studies showing a relationship between cognitive function and MR measures of brain atrophy. $7,8,22,28$ This discrepancy may be due to the fact that the number of subjects examined in this study is low. The presence of attention deficits early in the disease course of our patients, partly attributable to CAT sensitivity, may also contribute to the absence of correlation. A longitudinal study in individual patients $^{6}$ or a larger cohort group is required to determine whether attention deficits and brain atrophy correlate in Japanese MS patients with minor brain lesion loads.

\section{Conclusion}

Japanese relapsing-remitting MS patients with minor brain lesion loads frequently have attention deficits beginning in the initial phase of their disease. Their attention deficits are characterized by a slowness of automatic information processing, which spares controlled information processing. A correlation between cognitive functioning and brain atrophy was not found in this transverse study of a small cohort.

\section{Acknowledgments}

This study was supported by a grant from the Tamagawa University Center of Excellence from the Ministry of Education, Culture, Sports, Science and Technology (MEXT) and the Core Research for Evolutionary Science and Technology (CREST; no 17022035) and by a Grant-in-Aid for Scientific Research on Priority Areas-System Study on Higher-order Brain Functions from MEXT (no 20020026). This study was also supported in part by a Showa University Grant-in-Aid for Innovative Collaborative Research Projects and a Special Research Grant-in-Aid for Development of Characteristic Education from MEXT.

\section{Disclosure}

The authors report no conflicts of interest in this work.

\section{References}

1. Rao SM, Leo GJ, Bernardin L, Unverzagt F. Cognitive dysfunction in multiple sclerosis. I. Frequency, patterns, and prediction. Neurology. 1991;41(5):685-691.

2. Rao SM, Leo GJ, Ellington L, Nauertz T, Bernardin L, Unverzagt F. Cognitive dysfunction in multiple sclerosis. II. Impact on employment and social functioning. Neurology. 1991;41(5):692-696. 
3. Schulz D, Kopp B, Kunkel A, Faiss JH. Cognition in the early stage of multiple sclerosis. J Neurol. 2006;253(8):1002-1010.

4. Filley CM, Heaton RK, Nelson LM, Burks JS, Franklin GM. A comparison of dementia in Alzheimer's disease and multiple sclerosis. Arch Neurol. 1989;46(2):157-161.

5. Kujala P, Portin R, Revonsuo A, Ruutiainen J. Automatic and controlled information processing in multiple sclerosis. Brain. 1994;117(Pt 5): 1115-1126.

6. Zivadinov R, Sepcic J, Nasuelli D, et al. A longitudinal study of brain atrophy and cognitive disturbances in the early phase of relapsing-remitting multiple sclerosis. J Neurol Neurosurg Psychiatry. 2001;70(6):773-780.

7. Benedict RH, Weinstock-Guttman B, Fishman I, Sharma J, Tjoa CW, Bakshi R. Prediction of neuropsychological impairment in multiple sclerosis: comparison of conventional magnetic resonance imaging measures of atrophy and lesion burden. Arch Neurol. 2004;61(2): 226-230.

8. Sánchez MP, Nieto A, Barroso J, Martín V, Hernández MA. Brain atrophy as a marker of cognitive impairment in mildly disabling relapsingremitting multiple sclerosis. Eur J Neurol. 2008;15(10):1091-1099.

9. Shibasaki H, McDonald WI, Kuroiwa Y. Racial modification of clinical picture of multiple sclerosis: comparison between British and Japanese patients. J Neurol Sci. 1981;49(2):253-271.

10. Poser CM, Paty DW, Scheinberg L, et al. New diagnostic criteria for multiple sclerosis: guidelines for research protocols. Ann Neurol. 1983; 13(3):227-231.

11. Kurtzke JF. Rating neurologic impairment in multiple sclerosis: an expanded disability status scale (EDSS). Neurology. 1983;33(11): 1444-1452.

12. Wingerchuk DM, Lennon VA, Pittock SJ, Lucchinetti CF, Weinshenker BG. Revised diagnostic criteria for neuromyelitis optica. Neurology. 2006;66(10):1485-1489.

13. Japan Society for Higher Brain Dysfunction. Clinical Assessment for Attention. Tokyo, Japan: Shinko-Igaku Press; 2006.

14. Lezak MD. Neurological Assessment. 3rd ed. New York: Oxford University Press; 2004.

15. Mizuno M. Neuropsychological characteristics of right hemisphere damage: investigation by attention tests, concept formation and change test, and self-evaluation task. Keio J Med. 1991;40(4):221-234.
16. Smith A. The symbol digit modalities test: a neuropsychological test for economic screening of learning and other cerebral disorders. Learn Disord. 1968;3:83-91.

17. Morris N, Jones DM. Memory updating in working memory: the role of the central executive. Br J Psychol. 1990;81(2):111-121.

18. Gronwall D, Wrightson P. Delayed recovery of intellectual function after minor head injury. Lancet. 1974;2(7881):605-609.

19. Sohlberg MM, Mateer CA. Effectiveness of an attention training program. J Clin Exp Neuropsychol. 1987;9(2):117-130.

20. Beck LH, Bransome ED Jr, Mirsky AF, Rosvold HE, Sarason I. A continuous performance test of brain damage. $J$ Consult Psychol. 1956;20(5):343-350.

21. Lazeron RH, Boringa JB, Schouten M, et al. Brain atrophy and lesion load as explaining parameters for cognitive impairment in multiple sclerosis. Mult Scler. 2005;11(5):524-531.

22. Lazeron RH, de Sonneville LM, Scheltens P, Polman CH, Barkhof F Cognitive slowing in multiple sclerosis is strongly associated with brain volume reduction. Mult Scler. 2006;12(6):760-768.

23. Bermel RA, Bakshi R, Tjoa C, Puli SR, Jacobs L. Bicaudate ratio as a magnetic resonance imaging marker of brain atrophy in multiple sclerosis. Arch Neurol. 2002;59(2):275-280.

24. Stoquart-Elsankari S, Bottin C, Roussel-Pieronne M, Godefroy O. Motor and cognitive slowing in multiple sclerosis: an attentional deficit? Clin Neurol Neurosurg. 2010;112(3):226-232.

25. Godefroy O, Lhullier-Lamy C, Rousseaux M. SRT lengthening: role of an alertness deficit in frontal damaged patients. Neuropsychologia. 2002;40(13):2234-2241.

26. Houghton G, Tipper SP. A model of inhibitory mechanisms in selective attention. In: Dagenbach D, Carr TH, editors. Inhibitory Processes in Attention, Memory and Language. San Diego, CA: Academic Press; 1994.

27. Cifelli A, Arridge M, Jezzard P, Esiri MM, Palace J, Matthews PM. Thalamic neurodegeneration in multiple sclerosis. Ann Neurol. 2002; 52(5):650-653.

28. Houtchens MK, Benedict RH, Killiany R, et al. Thalamic atrophy and cognition in multiple sclerosis. Neurology. 2007;69(12):1213-1223.
Neuropsychiatric Disease and Treatment

\section{Publish your work in this journal}

Neuropsychiatric Disease and Treatment is an international, peerreviewed journal of clinical therapeutics and pharmacology focusing on concise rapid reporting of clinical or pre-clinical studies on a range of neuropsychiatric and neurological disorders. This journa is indexed on PubMed Central, the 'PsycINFO' database and CAS

\section{Dovepress}

The manuscript management system is completely online and includes a very quick and fair peer-review system, which is all easy to use. Visit http://www.dovepress.com/testimonials.php to read real quotes from published authors. 